\title{
Pivotality of Nodes in Reachability Problems Using Avoidance and Transit Hitting Time Metrics
}

\author{
Golshan Golnari, Dan Boley, \\ Computer Science \& Engineering \\ University of Minnesota \\ Minneapolis, MN, USA \\ [golnari, boley]@cs.umn.edu
}

\author{
Yanhua Li, and Zhi-Li Zhang \\ Computer Science \& Engineering \\ University of Minnesota \\ Minneapolis, MN, USA \\ [yanhua, zhzhang]@cs.umn.edu
}

\begin{abstract}
Reachability is crucial to many network operations in various complex networks. More often than not, however, it is not sufficient simply to know whether a source node $s$ can reach a target node $t$ in the network. Additional information associated with reachability such as how long or how many possible ways node $s$ may take to reach node $t$. In this paper we analyze another piece of important information associated with reachability - which we call pivotality. Pivotality captures how pivotal a role that a node $k$ or a subset of nodes $S$ may play in the reachability from node $s$ to node $t$ in a given network. We propose two important metrics, the avoidance and transit hitting times, which extend and generalize the classical notion of hitting times. We show these metrics can be computed from the fundamental matrices associated with the appropriately defined random walk transition probability matrices and prove that the classical hitting time from a source to a target can be decomposed into the avoidance and transit hitting times with respect to any third node. Through simulated and real-world network examples, we demonstrate that these metrics provide a powerful ranking tool for the nodes based on their pivotality in the reachability.
\end{abstract}

\section{Categories and Subject Descriptors}

G.2.2 [Graph Theory]: Network problems, Graph algorithms

\section{Keywords}

Reachability; Pivotality; Hitting time

\section{INTRODUCTION}

Reachability is crucial in any type of complex networks, be them communication and computer networks, power grids, transportation networks or social networks [15][8][12]. More often than not, however, it is not sufficient simply to know that a node $s$ can reach another node $t$ in the network. Additional information associated with reachability such as how long (e.g., in terms of number of intermediate nodes to be traversed or some other measures of time or cost) or how many possible ways (e.g., in terms of paths) for node $s$ to reach node $t$. Such information is essential for selecting

Copyright is held by the International World Wide Web Conference Committee (IW3C2). IW3C2 reserves the right to provide a hyperlink to the author's site if the Material is used in electronic media. WWW 2015 Companion, May 18-22, 2015, Florence, Italy. ACM 978-1-4503-3473-0/15/05.

http://dx.doi.org/10.1145/2740908.2744713. paths for packet routing or information/commodity delivery, flow scheduling, power management, traffic control, load balancing and so forth in communication and computer networks, power grids and transportation networks. In social networks, such information provides a measure of the strength of social ties between nodes and the role of nodes in these networks.

The shortest path distance is a commonly used metric to measure the reachability from a source $s$ to a target $t$. Its drawback lies in that it fails to capture the "path diversity" between the two nodes. Information about path diversity is important for load balancing, resilient routing and other network operations, and more broadly, for analyzing the overall network robustness. Path diversity can perhaps be best captured via random walks on the (weighted, directed or undirected) graph associated with the underlying network: The hitting time (sometimes referred to as the random walk distance), $H_{s t}$, measures the expected numbers of steps it takes for a random walker starting from node $s$ to reach node $t$ for the first time. This metric is in general asymmetric, $H_{s t} \neq H_{t s}$, even the underlying graph is undirected. The (symmetric) commute time, defined as $C_{s t}=H_{s t}+H_{t s}$, is also known as the effective resistance distance due to its connection to electric resistive networks [3].

In this paper, we analyze another piece of important information associated with reachability - which we call pivotality. Pivotality captures how pivotal a role that a third node $k$ or a subset of nodes $S$ may play in the reachability from node $s$ to node $t$ in a given network by quantifying how many (and how long) paths from $s$ to $t$ go through $k$ or $S$, and how many do not. Such information is critical for load balancing, protection routing, fast rerouting, robustness analysis and other network operations in various types of complex networks. For example, $k$ can represent a "middlebox" (e.g., firewall) and $S$ a set of application proxy servers in the cloud; one would like to quantify the overhead incurred for routing traffic from $s$ via $k$ or $S$ to $t$. Likewise, $k$ or $S$ can represent a single or multiple congested or failed nodes, or potentially compromised, vulnerable or untrustworthy nodes; one would like to quantify the overhead incurred for routing traffic from $s$ to $t$ by completely bypassing $k$ or $S$.

For these purposes, we introduce two inter-related metrics: avoidance and transit hitting times which extends and generalizes the classical notion of hitting times: the avoidance hitting time from a node $s$ to another node $t$ with respect to $k$ or $S$ is the expected number of steps it takes for a random walker starting from node $s$ to first hit node $t$ without ever hitting node $k$ or any node in $S$; whereas the transit hitting time from a node $s$ to another node $t$ with respect to $k$ or $S$ is the expected number of steps it takes for a random walker starting from node $s$ to first hit node $k$ or any node in $S$ and then hit node $t$ for the first time. We establish that both avoidance and transit hitting times can be computed from the fundamental matrices 
associated with the appropriately defined random walk transition probability matrices. We further show that with respect to any third node $k$, hitting time $H_{s t}$ can be decomposed into the avoidance hitting time and transit hitting time with respect to $k$. By exploiting relationships between the hitting time and avoidance/transit hitting times, we quantify the role and impact of node $k$ in the connectivity/reachability from node $s$ to $t$. Finally we use several simulated and real-world network examples to illustrate the advantages and utility of avoidance and transit hitting times, especially in comparison with existing metrics proposed in the literature.

\section{RELATED WORK}

The notions of centrality and betweenness have been widely studied in the literature to capture various roles that nodes play in social and other complex networks. These metrics are commonly defined in terms of the shortest paths between pairs of nodes. For example, in social network analysis the (shortest-path) centrality of a node $s$ is the average (shortest-path) distance from node $s$ to all other nodes in the network. Freeman's well-known (shortest-path) $b e$ tweenness [6] counts the number of shortest paths (from any source $s$ to any target $t$ ) that pass through a given node $k$. Hence larger the betweenness value of a node $k$, more vital it is to the reachability to many pairs of nodes in the network. As mentioned earlier, a main drawback of metrics defined solely in terms of shortest paths is that it does not capture the path diversity in the network. For this reason, various centrality measures based on random walks on graphs have been proposed in the literature. By exploiting the connection between random walks and current flows in electrical resistive networks [3], Newman [11] proposes a random-walk betweenness (or current flow betweenness) metric which measures the total sum of current flows passing through a given node $k$ from all sources to all sinks ("targets") in a network. Fouss et al. [5] use the hitting time (and commute time) associated with random walks on graphs as the measure of similarity between nodes in a recommendation system application. Sarkar et al. [14] develop a fast proximity search in large graphs by means of hitting time. Chen et al. [2] present a clustering algorithm via hitting time on directed graphs. All of these studies are confined to applying the classical hitting time to solving graph/network problems.

Closely related to what we study in this paper, Ranjan and Zhang [13] introduce the notion of (forced) detour cost of a random walker from a source $s$ to a target $t$ with respect to a third node $k$, which is defined as $\Delta H_{s t}^{(k)}:=H_{s k}+H_{k t}-H_{s t}$. Namely, the (forced) detour cost is the additional steps incurred when a random walker starts at source node $s$ and is forced to first visit the third node $k$, and then starts from node $k$ to reach target node $t$ vs. the number of the steps it takes starting at source node $s$ and hitting target node $t$ for the first time. Ranjan and Zhang show [13] that aggregated over all pairs of sources and targets, $\sum_{s} \sum_{t} \Delta H_{s t}^{(k)}=L_{k k}^{+}$. Here $L_{k k}^{+}$is the diagonal entries of $L^{+}$, the Penrose-Moore pseudo-inverse of the graph Laplacian $L=D-A$, where $A=\left[a_{i j}\right]$ is the adjacency matrix of a graph (network) and $D=\operatorname{diag}\left[d_{i}\right], d_{i}=\sum_{j} a_{i j}$, is the diagonal degree matrix. Based on this (forced) detour cost as well as several other interpretations of the diagonal entries $L_{k k}^{+}$of $L^{+}$, Ranjan and Zhang advocate $C^{*}(k):=1 / L_{k k}^{+}$as a new node centrality measure - referred to as the structural or topological centrality, and demonstrate that $C^{*}(k):=1 / L_{k k}^{+}$indeed better captures the structural/topological roles that node $k$ plays in a network than existing centrality metrics, in particular in terms of their roles in the overall network robustness. Motivated by the results in [13], in this paper we aim to provide a more precise characterization of how pivotal a role a third node $k$ may play in the random walks from a source
Table 1: List of Notations

\begin{tabular}{|l|l|}
\hline$F^{(j)}$ & Fundamental matrix for target $j$ \\
$F_{i m}^{(j)}$ & The $i m$-th entry of Fundamental matrix $F^{(j)}$. \\
& $\begin{array}{l}\text { It represents the expected number of passages through } \\
\text { node } m, \text { starting from } i \text { and before hitting } j\end{array}$ \\
$F^{(S)}$ & Fundamental matrix for a target set $S$, say, $S=\{j, k\}$ \\
$F_{i m}^{(S)}$ & The $i m$-th entry of the fundamental matrix $F^{(S)}$ \\
$Q_{i j}^{(\bar{k})}$ & The probability of hitting $j$ before $k$ when starting from $i$ \\
$H_{i j}$ & Hitting time from $i$ to $j$. It is $i j$-th entry of the hitting time matrix $H$ \\
$H_{i, S}$ & Hitting time from $i$ to a target set $S$, say, $S=\{j, k\}$ \\
$H_{i j}^{(\bar{k})}$ & Avoidance hitting time from $i$ to $j$ conditioned on avoiding $k$ \\
$H_{i j}^{(\breve{k})}$ & Transit hitting time from $i$ to $j$ conditioned on transiting $k$ \\
\hline
\end{tabular}

node $s$ to a target node $t$ by probabilistically quantifying the number of paths from source $s$ to target $t$ that circumvent node $k$ vs. those that traverse node $k$ that the random walker is likely to take. This leads us to introduce two inter-related metrics, avoidance and transit hitting times, to measure the pivotality of node $k$ in the random walks from source $s$ to target $t$.

\section{THEORY OF AVOIDANCE AND TRAN- SIT HITTING TIMES}

In this section, we first provide an overview of the existing theory on random walks on a general weighted and directed networks and introduce the classical notions of hitting and commute times. We then define the new notions of avoidance and transit hitting time metrics and demonstrate how they can be computed. The section culminates with the Hitting Time Decomposition Theorem.

\subsection{Background and Notations}

A network can be abstractly modeled as a weighted and directed graph, denoted by $G=(V, E, A)$. Here $V$ is the set of nodes in the network such as routers or switches in a communication network or users in a social network; $E$ is the set of (directed) edges representing the (physical or logical) connections between nodes (e.g., a communication link from a node $i$ to a node $j$ ) or entity relations (e.g., follower-followee relation between two users). The affinity (or adjacency) matrix $A=\left[a_{i j}\right]$ is assumed to be nonnegative, i.e., $a_{i j} \geq 0$, where $a_{i j}>0$ if and only if $\langle i, j\rangle \in E$. We remark that here $a_{i j}$ captures certain "affinity" or "closeness" from node $i$ to node $j$, i.e., the communication capacity from node $i$ to node $j$, or strength of social tie between node $i$ and node $j$. Hence larger $a_{i j}$ is, "closer" node $i$ is to node $j$-in other words, $A$ can be viewed as a "similarity" matrix. This is in contrast to a "cost" or "distance" matrix $C=\left[c_{i j}\right]$ that is sometimes used to denote link weights, where larger $c_{i j}$ is, "farther away" (or "dissimilar") node $i$ is from node $j$. (One can easily convert a cost matrix $C$ to an affinity matrix $A$, e.g., by setting $a_{i j}=1 / c_{i j}$.) Throughout the paper, we assume that networks under consideration are strongly connected, namely, there is at least one path from any node $i$ to any other node $j$.

Given $G$, a random walk on $G$ is a Markov chain on $G$ given by the transition probability matrix $P=D^{-1} A$, where $D=\operatorname{diag}\left[d_{i}\right]$ and $d_{i}=\sum_{j} a_{i j}$ ( $d_{i}$ is often referred to as the (out-)degree of node $i$ and $D$ is the (diagonal) degree matrix). Given a random walk starting at node $s$, at each step $i$ the random walker picks an out-going edge randomly with probability $P_{i j}=\frac{a_{i j}}{\sum_{j} a_{i j}}$. The hitting time $H_{s t}$ is defined as the expected time (or number of steps) it takes for a random walk starting at node $s$ to reach ("hit") node $t$ for the first time. Intuitively, the larger $H_{s t}$ is, the farther node $t$ is located from node $s$. Hitting times can be computed directly using the fundamental ma- 
$\operatorname{trix} F$ [3] for absorbing Markov chains. Let $n=|V|$. For each $j \in V$, we use $P_{\mid j}$ to denote the $(n-1) \times(n-1)$ sub-matrix after deleting the row and column $j$ from the transition probability matrix $P$. Then the fundamental matrix $F^{(j)}$ is defined as $F^{(j)}:=\left(I-P_{\mid j}\right)^{-1}$. It can be proved that the $i k$-th entry of the matrix $F^{(j)}, F_{i k}^{(j)}$, yields the expected number of passages through node $k$ for a random walk starting at node $i$ and before hitting $j$. Then the hitting time from source $i$ to target $j$ is given by $H_{i j}=\sum_{m} F_{i m}^{(j)}$, namely the sum over the expected number of passages through all the other nodes before hitting the target [3]. More generally, we can define the hitting time from a source node $i$ to a set of target nodes $S$, denoted by $H_{i, S} . H_{i, S}$ is defined as the expected number of steps for a random walk starting at node $i$ and hitting one of the nodes in $S$ for the first time. For example, for $S=\{j, k\}, H_{i,\{j, k\}}$ is the expected number of steps for a random walk starting at node $i$ and hitting either $j$ or $k$ for the first time. Again we can compute $H_{i, S}$ using an appropriately defined fundamental matrix. Let $P_{\mid S}$ denote the $(n-|S|) \times(n-|S|)$ submatrix after deleting the rows and columns corresponding to the indices $j \in S$ from $P$, and define $F^{(S)}:=\left(I-P_{\mid S}\right)^{-1}$. Hence $H_{i, S}=\sum_{m} F_{i m}^{(S)}$. Table 1 summarizes some of the notations used in this paper.

\subsection{Avoidance and Transit Hitting Times}

We are now in position to introduce two inter-related metrics, avoidance and transit hitting times by extending and generalizing the classical notion of hitting times. Consider a target set $S$ consisting of two target nodes $j$ and $k$. Recall that the hitting time $H_{i, S}=H_{i,\{j, k\}}$ is the expected number of steps for a random walk starting at node $i$ to first hit either target $j$ or target $k$. Since both target nodes $j$ and $k$ are absorbing, there are only two possible ways for the random walk to hit the target set $S=\{j, k\}$ : either the random walk first hits node $j$ without ever hitting node $k$ or it first hits node $k$ without ever hitting node $j$. We use $Q_{i j}^{(\bar{k})}$ to denote the probability that the random walk first hits target node $j$. It is not too hard to see that $Q_{i j}^{(\bar{k})}=\sum_{m} F_{i m}^{(\{j, k\})} P_{m j}$. Then the probability that the random walk first hits target node $k$ is then given by $Q_{i k}^{(\bar{j})}=1-Q_{i j}^{(\bar{k})}=\sum_{m} F_{i m}^{(\{j, k\})} P_{m k}$. Using these relations, we can analyze the (random) paths taken by a random walk starting at node $i$ and first hitting target node $j$ while avoiding a particular third node $k$ and quantify the probability such paths are taken by the random walker. This leads us to define the notion of avoidance hitting time. For any third node $k$, the avoidance hitting time $H_{i j}^{(\bar{k})}$ is the expected number of steps taken by a random walk which starts at node $i$ and hits node $j$ for the first time while without ever hitting node $k$ in between (i.e., by always avoiding node $k$ on its way to target node $j$ ). We have the following theorem, the proof of which is delegated to the Appendix.

THEOREM 1. (Avoidance Hitting Time)

Hitting time from node $i$ to node $j$ conditioned on avoiding node $k$ can be computed using the following equation:

$$
H_{i j}^{(\bar{k})}=\frac{\sum_{m} F_{i m}^{(\{k, j\})} Q_{m j}^{(\bar{k})}}{Q_{i j}^{(\bar{k})}} .
$$

Closely related to the avoidance hitting time is the notion of transit hitting time. For any third node $k$, the transit hitting time $H_{i j}^{(\breve{k})}$ is the expected number of steps taken by a random walk which starts at node $i$ and always traverse node $k$ before hits target node $j$ for the first time. Using the avoidance hitting time, we can express $H_{i j}^{(\breve{k})}$ as

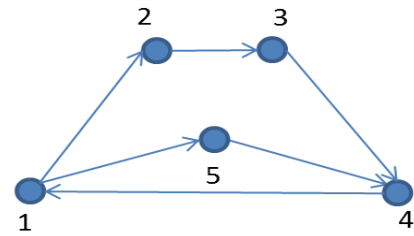

Figure 1: Network Example 1

follows:

$$
H_{i j}^{(\breve{k})}=H_{i k}^{(\bar{j})}+H_{k j}
$$

Using the avoidance and transit hitting times, we can now divide the paths (or walks) between a source node $i$ to a target node $j$ into two groups with respect to an arbitrary third node $k$ : those paths that exclude or avoid node $k$ and those that include or transit node $k$. The probability that a random walk takes either a path/walk from the first group vs. that from the second group is given by $Q_{i j}^{(\bar{k})}$ and $Q_{i k}^{(\bar{j})}$. This yields the following Hitting Time Decomposition Theorem, the proof of which can be found in the Appendix.

THEOREM 2. (Hitting Time Decomposition)

The hitting time from node $i$ to node $j$ can be decomposed into an "avoidance" hitting time component and a "transit" hitting time component with respect to any node $k$ as follows:

$$
H_{i j}=Q_{i j}^{(\bar{k})} H_{i j}^{(\bar{k})}+Q_{i k}^{(\bar{j})} H_{i j}^{(\breve{k})} .
$$

The transit and avoidance hitting times can be generalized to an arbitrary (sub)set of nodes, $H_{i j}^{\left(\check{S}_{1}\right)}, H_{i j}^{\left(\bar{S}_{2}\right)}$, and combined, $H_{i j}^{\left(\check{S}_{1}, \bar{S}_{2}\right)}$, where the last term represents the hitting time from node $i$ to node $j$ conditioned on traversing any node in $S_{1}$ while avoiding all nodes in $S_{2}$. Due to space limitation, we will not elaborate here; more details can be found in [7].

\section{NODE PIVOTALITY IN NETWORK REACH- ABILITY}

In this section we examine and quantify how pivotal a role a node $k$ plays in reachability from a source node $s$ to a target node $t$ using our proposed avoidance and transit hitting metrics. In particular, we propose the avoidance-transit hitting time pivotality metric (ATH). For a given node $k$ with respect to a pair of source and target nodes $s$ and $t$, it is defined as follows:

$$
e_{A T H}(k)=H_{s t}-H_{s t}^{(\check{k})}=H_{s t}-\left(H_{s k}^{(\bar{t})}+H_{k t}\right) .
$$

Note that if all paths from node $s$ to node $t$ go through a node $k^{*}$, then $e_{A T H}\left(k^{*}\right)=0$. In this case, $k^{*}$ is the most "pivotal" point of any path from $s$ to $t$ in that all paths rely on $k^{*}$. We claim that in such a case, for any other node $k, e_{A T H}(k) \leq 0$; due to space limitation, we will omit the proof here. In general, $e_{A T H}(k)$ can be either positive, indicating that paths going through node $k$ are overall shorter than an "average" path from node $s$ to node $t$; or negative, indicating that paths going through node $k$ are overall longer that an "average" path from node $s$ to node $t$.

For comparison, we also consider other metrics proposed in the literature. We define the shortest-path pivotality metric (SHP) to measure the pivotality of node $k$ using the shortest paths only: $e_{S H P}(k)=$ $s h p_{s t}-\left(s h p_{s k}+s h p_{k t}\right)$. The maximum flow pivotality metric (MF), $e_{M F}(k)$, measures the amount of the maximum flow from $s$ to $t$ that goes through node $k$ in a flow network, where the weight of edges 


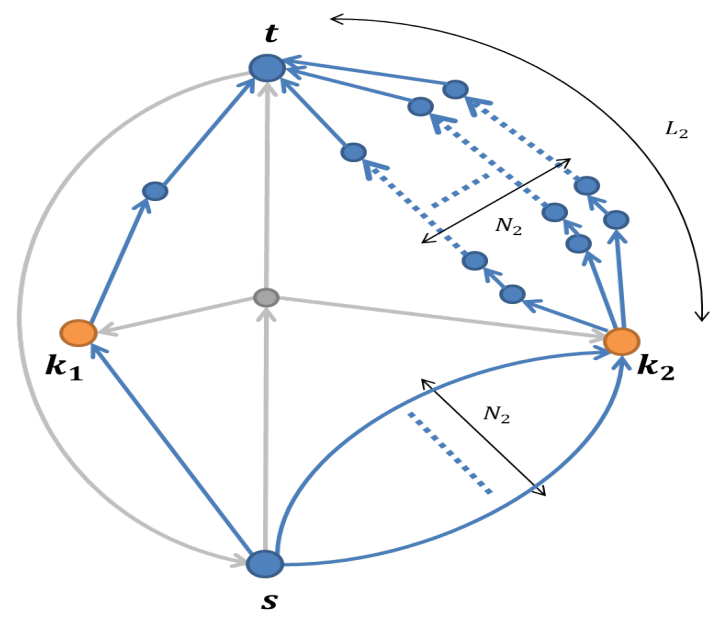

Figure 2: Network Example 2

indicate their capacity. The (classical) hitting time pivotality metric $(\mathrm{CH})$ is defined as the negative of the (forced) detour cost defined in [13],

$$
e_{C H}(k):=-\Delta H_{s t}^{(k)}=H_{s t}-\left(H_{s k}+H_{k t}\right) .
$$

Notice the similarity between $e_{A T H}(k)$ and $e_{C H}(k)$, except the terms $H_{s k}^{(\bar{t})}$ and $H_{s k}$. Due to the triangle inequality of the shortest path distance and the hitting time, $e_{S H P}(k) \leq 0$ and $e_{C H}(k) \leq 0$ whereas by definition, $e_{M F}(k) \geq 0$ for all $k$ and all pairs of source and target nodes, $s$ and $t$. Despite these differences, in terms of ranking of nodes based on their pivotality using each metric, what matters is their relative values: as long as $e\left(k_{1}\right)<e\left(k_{2}\right)$, node $k_{2}$ is more "pivotal" than $k_{1}$ in terms of reachability from $s$ to $t$.

\subsection{Understanding Pivotality Metrics: Exam- ples}

Using several simple network examples, in this section we illustrate and compare the behavior of the pivotality metrics defined above. First consider the simple network example shown in Fig. 1 where the weight of all edges is 1 , i.e., $a_{i j}=1$. With node 1 being the source and node 4 the target, it is intuitively apparent that node 5 is more "pivotal" than node 2 or node 3 , given that it is on the shorter path. The pivotaliy metrics computed using the four methods are shown in Table 2. We say that both the MF and $\mathrm{CH}$ metrics fail to rank the nodes correctly in that they are not able to recognize the higher pivotality of node 5 over nodes 2 and 3 .

Figure 2 provides a more general network example which can help illustrate the different behaviors of the pivotality metrics under study. In this network, there exists a shortest path of length 2 from source $s$ to target $t$ (gray-colored path) interconnected to two groups of (blue-colored) paths passing through $k_{1}$ and $k_{2}$ : a threehop path from source $s$ via node $k_{1}$ to target $t$, whereas there are $N_{2}$ parallel paths going through node $k_{2}$, the length of which are $L_{2}+1$. If $L_{2}=2$ and $N_{2}=1$ the network is symmetric with respect to $k_{1}$ and $k_{2}$ and yields equal pivotality for $k_{1}$ and $k_{2}$ in reachability from $s$ to $t$ (second row of Table 3). However, if $N_{2} \approx 1$ and $L_{2} \gg 2$, intuitively node $k_{1}$ plays a more pivotal role than $k_{2}$. On the other hand, as the number $N_{2}$ of parallel paths going through $k_{2}$ increases while their length $L_{2}+1$ is not significantly much longer than 3 , say, $L_{2}=3$, node $k_{2}$ will play an increasingly more pivotal role in delivering traffic, information or other commodity from node $s$ to node $t$. Intuitively, there is a trade-off between $N_{2}$ and $L_{2}$ : more parallel paths going through node $k_{2}$ will increase its pivotal-

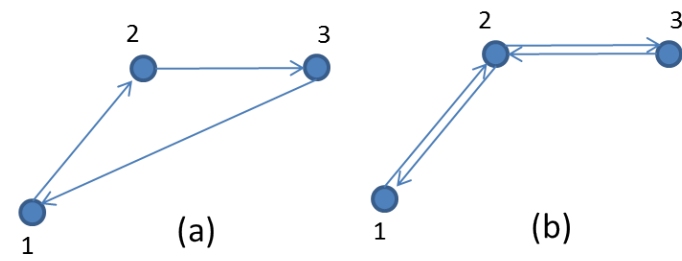

Figure 3: Network example 3

Table 2: Pivotality metrics in Network Example 1: source node 1 and target node 4

\begin{tabular}{c|ccc} 
nodes & 2 & 3 & 5 \\
\hline$e_{S H P}$ & -1 & -1 & 0 \\
$e_{M F}$ & 0.5 & 0.5 & 0.5 \\
$e_{C H}$ & -3.5 & -3.5 & -3.5 \\
$e_{A T H}$ & -0.5 & -0.5 & 0.5
\end{tabular}

ity as it enhances the overall "capacity" from node $s$ to node $t$; however larger $L_{2}$ will diminish its pivotality as longer paths increase the "cost" of using these parallel paths. Despite such intuitions regarding the relative pivotality values of node $k_{1}$ and node $k_{2}$, if $L_{2}>2$ the SHP pivotality metric will always rank node $k_{1}$ higher than $k_{2}$ independently of $N_{2}$ (for $L_{2}=2$ gives the same ranking to them). Whereas, as long as $N_{2}>1$, the MF pivotality metric will always rank node $k_{2}$ higher than node $k_{1}$ independently of $L_{2}$. Hence both these two metrics fail to capture the differing roles of node $k_{2}$ with varying $N_{2}$ and $L_{2}$. To evaluate the performance of $\mathrm{CH}$ and $\mathrm{ATH}$ pivotality metrics in capturing the differing roles of node $k_{2}$ with varying $N_{2}$ and $L_{2}$, some example values are shown in Table 3. Based on these results, the $\mathrm{CH}$ pivotality metric ranks node $k_{2}$ higher than node $k_{1}$ as long as $N_{2}>1$, and ranks them the same when $N_{2}=1$ no matter how large is $L_{2}$, behaving the same as the MF pivotality metric. However, the ATH pivotality metric ranks successfully node $k_{1}$ higher than node $k_{2}$ when $N_{2}$ is close to 1 and $L_{2}$ is quite larger than 2 .

The subtle difference in the behaviors of the $\mathrm{CH}$ and ATH pivotality metrics lies in the term $H_{s k}$ in eq.(5) vs. the term $H_{s k}^{(\bar{t})}$ in eq.(4). Namely, in accounting for the (forced) detour cost, the $\mathrm{CH}$ method allows and includes paths/walks from the source node $s$ to the third node $k$ that may have already traversed the target node $t$; in network example 2, increasing $L_{2}$ has a destructive effect on the $\mathrm{CH}$ pivotality metric of $k_{1}$ by accounting the paths passing through $t$ before hitting $k_{1}$, such as the walk $\left(s-k_{2}-t-s-k_{2}-t-\ldots-s-k_{1}\right)$, and increasing the term $H_{s k_{1}}$ in eq.(5) as the result. In contrast, the ATH method excludes such paths/walks in accounting for the detour cost. As a result, the ATH provides a more precise quantification of the detour cost when a random walker is "forced" to transit a third node $k$, and thereby how pivotal a role node $k$ plays in the reachability from a source to a target.

The ATH metric allows us to identify nodes that are "superfluous" with respect to the reachability of a source to a target. This can be best illustrated by the two simple examples shown in Fig. 3 . In both examples, consider node 1 as the source and node 2 as the target. It is obvious that node 3 is "superfluous" with respect to this source-target pair in that node 3 plays no part in the reachability from node 1 to node 2 . In other words, if node 3 fails or is removed from the network, the reachability from node 1 to node 2 (and the associated "capacity") is not affected at all. This can be captured by the fact that in both networks in Figs. 3 (a) and (b), the 


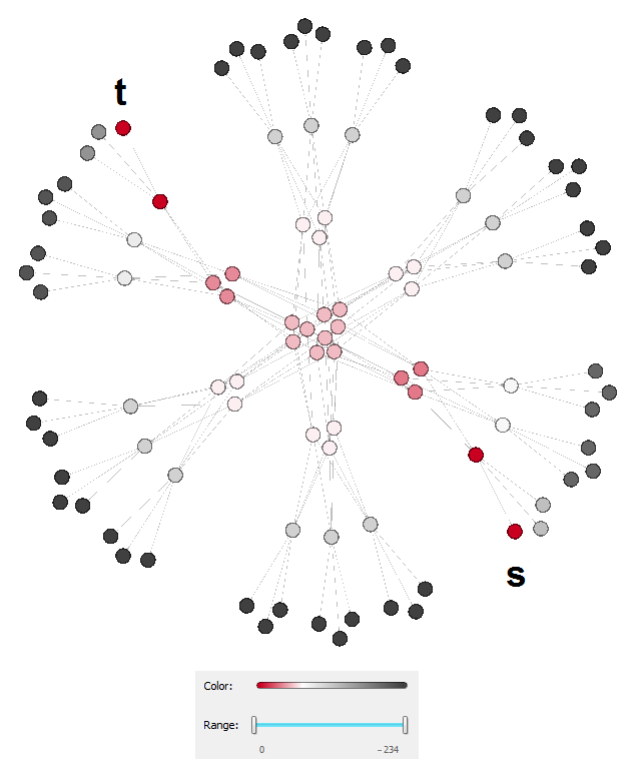

Figure 4: Node pivotality ranking in a Fat-tree network for the reachability of the source node $s$ to target node $t$ : red indicates highest pivotality and black shows non-pivotality.

Table 3: Pivotality metrics (CH and ATH only) in Network Example 2 for various choices of $N_{2}$ and $L_{2}$

\begin{tabular}{c|cc} 
& $e_{C H}$ & $e_{A T H}$ \\
& $k_{1}, k_{2}$ & $k_{1}, k_{2}$ \\
\hline$L_{2}=1, N_{2}=2$ & $-7,-2.5$ & $-0.75,0.36$ \\
$L_{2}=2, N_{2}=1$ & $-5.14,-5.14$ & $-0.14,-0.14$ \\
$L_{2}=2, N_{2}=2$ & $-8.17,-2.92$ & $-0.17,-0.06$ \\
$L_{2}=20, N_{2}=2$ & $-29.17,-10.42$ & $10.33,-7.56$ \\
$L_{2}=20, N_{2}=1$ & $-15.14,-15.14$ & $7.86,-10.14$
\end{tabular}

probability of hitting node 3 before node 2 is zero, i.e., $Q_{13}^{(\overline{2})}=0$. Thus the denominator of the term $H_{13}^{\overline{2}}$ in eq.(1) becomes zero and thus $H_{13}^{\overline{2}}=\infty$. This renders $e_{A T H}(3)=-\infty$ (see eq.(4)), indicating the non-pivotality of node 3 . In contrast, the $\mathrm{CH}$ metric and SHP metric yield $e_{C H}(3)=-3$ and $e_{S H P}(3)=-3$ for Fig. 3(a) and $e_{C H}(3)=-4$ and $e_{S H P}(3)=-2$ for Fig. 3(b) respectively.

\subsection{Node Pivotality Ranking using the ATH Metric}

Lastly we apply the node pivotality ranking using our ATH metric to two real-world networks: Fat-Tree [10] and the ESNet [4]. Fat-tree is a special $h$-ary $(h \geq 2)$ "tree-shaped" structure first proposed in [10] for efficient communication with uniform bi-section bandwidth, and for this reason it has been adopted in data center networks [1]. Fig. (4) shows 3-ary fat-tree structure with 99 nodes, where the node colors are shaded based on their ATH pivotality measures with respect to the reachability from the source $s$ to the target node $t$. In the figure, the color spectrum from red to white and then to black shows the range of the ATH value from high to low: the nodes with the larger ATH value, are more pivotal to the reachability from $s$ to $t$ are represented with red and "reddish" colors; in contrast, the nodes that play no part in the reachability from $s$ to $t$ are represented with black color. The results for the ESNet, the DoE energy science network with 68 nodes [4] are shown in

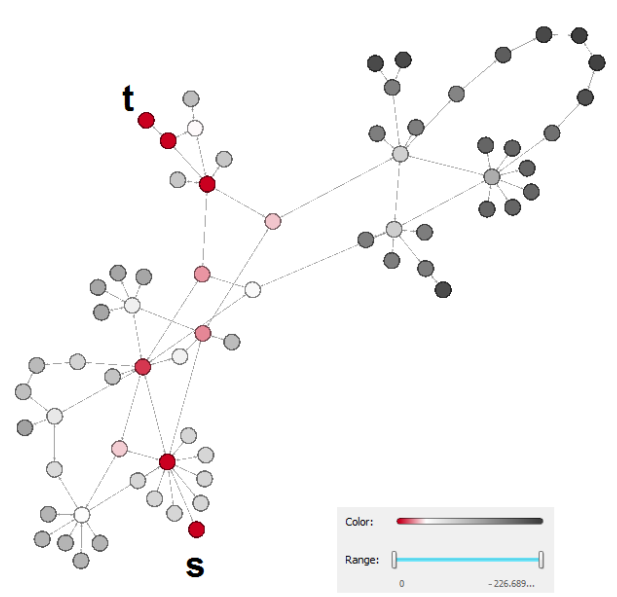

Figure 5: Node pivotality ranking in the ESNet network for the reachability of the source node $s$ to target node $t$ : red indicates highest pivotality and black shows non-pivotality.

Fig. (5). Both examples illustrate the efficacy of the ATH metric in correctly capturing and ranking the pivotality of nodes in the reachability from a source node to a target node. Due to space limitation, we do not elaborate on them.

\section{CONCLUSION}

In this paper we have extended and generalized the classical notion of hitting times to include the options of avoiding or transiting a specific node in random walks. We proved that the classical hitting time can be decomposed into avoidance hitting time and transit hitting time. We showed that these new metrics provide a powerful tool for assessing pivotality of nodes in the reachability of a source node to a target node. Intuitively, high pivotal nodes are the ones that make the reachability occur in shorter distance as they are traversed by a large of (shorter) paths for the reachability of source to target. Using some simple network examples, we compare our pivotality metric - the avoidance-transit hitting time (ATH) metric - with other metrics defined using the shortest paths, maximum flow or classical hitting time methods and demonstrated that these existing metrics fail to properly capture and assess the pivotality of nodes in the reachability from a source to a target while our ATH metric can. Finally, we applied the ATH method to two real-world network examples to rank the nodes based on their pivotality for the reachability from a source to a target. We visualized the results to demonstrate the performance of our proposed method.

Acknowledgment. This research was supported in part by DTRA grants HDTRA1-09-1-0050, HDTRA1-14-1-0040, DoD ARO MURI Award W911NF-12-1-0385, and NSF grants CNS-10171647, CRI-1305237 and IIS 1319749.

\section{REFERENCES}

[1] M. Al-Fares, A. Loukissas, and A. Vahdat. A scalable, commodity data center network architecture. In SIGCOMM. ACM New York, NY, USA, 2008.

[2] M. Chen, J. Liu, and X. Tang. Clustering via random walk hitting time on directed graphs. In $A A A I$, volume 8, pages 616-621, 2008. 
[3] P. G. Doyle and J. L. Snell. Random walks and electric networks. Carus mathematical monographs, 22, 2000.

[4] ESNet. Us energy science network. http: //www.es.net/.

[5] F. Fouss, A. Pirotte, J.-M. Renders, and M. Saerens. Random-walk computation of similarities between nodes of a graph with application to collaborative recommendation. Knowledge and data engineering, ieee transactions on, 19(3):355-369, 2007.

[6] L. C. Freeman. A set of measures of centrality based on betweenness. Sociometry, pages 35-41, 1977.

[7] G. Golnari and Z.-L. Zhang. Foundamental tensor, avoidance and transit hitting times and their applications, 2015. Research Manuscript in Preparation.

[8] A. R. Khakpour and A. X. Liu. Quantifying and querying network reachability. In Distributed Computing Systems (ICDCS), 2010 IEEE 30th International Conference on, pages 817-826. IEEE, 2010.

[9] C. K. Koc and G. Chen. Inversion of all principal submatrices of a matrix. Aerospace and Electronic Systems, IEEE Transactions on, 30(1):280-281, 1994.

[10] C. E. Leiserson. Fat-trees: universal networks for hardware-efficient supercomputing. Computers, IEEE Transactions on, 100(10):892-901, 1985.

[11] M. E. Newman. A measure of betweenness centrality based on random walks. Social networks, 27(1):39-54, 2005.

[12] M. Parandehgheibi and E. Modiano. Robustness of interdependent networks: The case of communication networks and the power grid. In Global Communications Conference (GLOBECOM), 2013 IEEE, pages 2164-2169.

[13] G. Ranjan and Z.-L. Zhang. Geometry of complex networks and topological centrality. Physica A: Statistical Mechanics and its Applications, 392(17):3833-3845, 2013.

[14] P. Sarkar, A. W. Moore, and A. Prakash. Fast incremental proximity search in large graphs. In Proceedings of the 25th international conference on Machine learning, pages 896-903. ACM, 2008.

[15] G. G. Xie, J. Zhan, D. A. Maltz, H. Zhang, A. Greenberg, G. Hjalmtysson, and J. Rexford. On static reachability analysis of ip networks. In INFOCOM 2005, volume 3, pages 2170-2183. IEEE, 2005.

\section{APPENDIX}

\section{Proof of Theorem 1:}

We divide the nodes into two groups: one consists of node $k$ and $j$, and the other one includes the rest. The first group is called absorbing $\mathcal{A}$ and the second one is transient $\mathcal{T}$. Reordering the nodes, transition probability matrix can be written as follows: $P=$ $\left[\begin{array}{cc}P_{\mathcal{T} \mathcal{T}} & P_{\mathcal{T} \mathcal{A}} \\ 0 & I_{\mathcal{A} \mathcal{A}}\end{array}\right]$. To obtain the conditional expected time from node $i$ to node $j$ given that not passing $k$ at all, we need to calculate the conditional probabilities of all possible paths that end in node $j$ and do not pass through $k$. In other words, for each path length $l$, where $l$ is an integer number, we calculate the conditional probability $\frac{\operatorname{Pr}_{l}(i, j)}{\sum_{l} \operatorname{Pr}_{l}(i, j)}$ of hitting node $i$ at the $l$-th step conditioned on hitting node $j$ sooner than node $k . \operatorname{Pr}_{l}(i, j)$ is the probability of the random walk being in one of the transient nodes in step 1 to $l-1$ and entering node $j$ at its $l$-th step. $\sum_{l} \operatorname{Pr}_{l}(i, j)$ is the total probability of hitting node $j$ sooner than node $k$.

$$
\operatorname{Pr}_{l}(i, j)=\left[P_{\mathcal{T} \mathcal{T}}^{l-1} P_{\mathcal{T} \mathcal{A}}\right](i, j)=e_{i} P_{\mathcal{T} \mathcal{T}}^{l-1} P_{\mathcal{T} \mathcal{A}}(:, j),
$$

where $e_{i}$ is a row vector of all zeros except its $i$-th entry which is equal to 1 . Note that $P_{\mathcal{T A}}(:, j)$ represents the column vector of transition probabilities from transient nodes to node $j$. So the conditional expected time from node $i$ to node $j$ avoiding node $k$ can be calculated as follows:

$$
H_{i j}^{(\bar{k})}=\frac{\sum_{l} l \operatorname{Pr}_{l}(i, j)}{\sum_{l} \operatorname{Pr}_{l}(i, j)}=\frac{\sum_{l} l\left[P_{\mathcal{T} \mathcal{T}}^{l-1} P_{\mathcal{T} \mathcal{A}}\right](i, j)}{\sum_{l}\left[P_{\mathcal{T} \mathcal{T}}^{l-1} P_{\mathcal{T} \mathcal{A}}\right](i, j)}
$$

The numerator can be re-written as follows:

$$
\begin{aligned}
\sum_{l} l\left[P_{\mathcal{T} \mathcal{T}}^{l-1} P_{\mathcal{T A}}\right](i, j) & =e_{i}\left(I+2 P_{\mathcal{T} \mathcal{T}}+3 P_{\mathcal{T} \mathcal{T}}^{2}+\ldots\right) P_{\mathcal{T} \mathcal{A}}(:, j) \\
& =e_{i}\left(I-P_{\mathcal{T} \mathcal{T}}\right)^{-2} P_{\mathcal{T} \mathcal{A}}(:, j)=e_{i} F^{2} P_{\mathcal{T} \mathcal{A}}(:, j) \\
& =e_{i} F Q^{(\bar{k})}=\sum_{m} F_{i m}^{(\{k, j\})} Q_{m j}^{(\bar{k})}
\end{aligned}
$$

Also, note that the denominator is simply the probability of absorption by node $j$ sooner than node $k$ starting from node $i$, which is equal to $Q_{i j}^{(\bar{k})}$ :

$$
\begin{aligned}
\sum_{l}\left[P_{\mathcal{T} \mathcal{T}}^{l-1} P_{\mathcal{T} \mathcal{A}}\right](i, j) & =e_{i}\left(I+P_{\mathcal{T} \mathcal{T}}+P_{\mathcal{T} \mathcal{T}}^{2}+\ldots\right) P_{\mathcal{T A}}(:, j) \\
& =e_{i}\left(I-P_{\mathcal{T} \mathcal{T}}\right)^{-1} P_{\mathcal{T} \mathcal{A}}(:, j)=e_{i} F P_{\mathcal{T A}}(:, j) \\
& =Q_{i j}^{(\bar{k})} .
\end{aligned}
$$

\section{Proof of Theorem 2:}

Based on Schur-complement theory and the relation of matrix inverse with its sub-matrix inverse [9], the following equation holds:

$$
F_{i m}^{(\{j, k\})}=F_{i m}^{(k)}-\frac{F_{i j}^{(k)} F_{j m}^{(k)}}{F_{j j}^{(k)}},
$$

where $\frac{F_{i j}^{(k)}}{F_{j j}^{(k)}}$ can be substituted by $Q_{i j}^{(\bar{k})}$ as follows:

$$
\begin{aligned}
Q_{i j}^{(\bar{k})} & =\sum_{m} F_{i m}^{(\{j, k\})} P_{m j}=\sum_{m}\left(F_{i m}^{(k)}-\frac{F_{i j}^{(k)} F_{j m}^{(k)}}{F_{j j}^{(k)}}\right) P_{m j} \\
& =\sum_{m} F_{i m}^{(k)} P_{m j}-\frac{F_{i j}^{(k)}}{F_{j j}^{(k)}} \sum_{m} F_{j m}^{(k)} P_{m j}=F_{i j}^{(k)}-\frac{F_{i j}^{(k)}}{F_{j j}^{(k)}}\left(F_{j j}^{(k)}-1\right) \\
& =\frac{F_{i j}^{(k)}}{F_{j j}^{(k)}}
\end{aligned}
$$

Taking sum over $m$ for both sides of Eq. (6), the following equation is obtained:

$$
H_{i,\{j, k\}}=H_{i k}-Q_{i j}^{(\bar{k})} H_{j k}
$$

On the other hand, we have the following relation based on the definition of the avoidance hitting time:

$$
H_{i,\{j, k\}}=Q_{i j}^{(\bar{k})} H_{i j}^{(\bar{k})}+Q_{i k}^{(\bar{j})} H_{i k}^{(\bar{j})}
$$

Eq. (7) and (8) together yield the following result:

$$
H_{i j}=Q_{i j}^{(\bar{k})} H_{i j}^{(\bar{k})}+Q_{i k}^{(\bar{j})}\left(H_{i k}^{(\bar{j})}+H_{k j}\right)=Q_{i j}^{(\bar{k})} H_{i j}^{(\bar{k})}+Q_{i k}^{(\bar{j})} H_{i j}^{(\check{k})},
$$

where $H_{i j}^{(\check{k})}=H_{i k}^{(\bar{j})}+H_{k j}$. 